\title{
Estilo parental e potencial criativo de crianças ${ }^{1}$
}

\section{Parenting style and creative potential of children}

\author{
Merav Dechaume* \\ Todd Lubart*
}

\begin{abstract}
RESUMO
Hoje em dia, as pessoas precisam ser capazes de pensar e produzir de forma criativa. Por isso, a estimulação das habilidades criativas constitui um objetivo importante para educadores e profissionais. O estilo parental que inclui práticas cotidianas de educação dos filhos, interações entre pais e filhos voltadas especificamente para a criatividade e percepções sobre a criatividade se relaciona fundamentalmente com o desenvolvimento do potencial criativo. Estes construtos moldam a compreensão das crianças sobre seu ambiente, permitindo-lhes formar uma mentalidade, qualidades pessoais, traços e habilidades que ajudam a determinar atitudes em relação à criatividade. Este estudo preliminar explorou a relação entre o estilo parental e o potencial criativo das crianças. Os pais participantes responderam aos questionários avaliando o nível de estrutura de rigidez nas práticas cotidianas de educação dos filhos, as interações entre pais e filhos especificamente voltadas para a criatividade e as percepções dos pais sobre a autoeficácia criativa de seus filhos. Seus filhos preencheram o instrumento de Avaliação do Potencial Criativo (EPoC), que mede o pensamento convergente e divergente nos domínios gráfico e verbal. Embora não tenhamos encontrado qualquer relação estatisticamente significativa entre o estilo parental e o potencial criativo, várias interações pai-filho se correlacionaram significativamente com as percepções dos pais. Quanto mais os pais incentivam a novidade, mais eles percebem que seus filhos demonstram comportamento criativo.
\end{abstract}

1 Traduzido por David Harrad. E-mail: davidharrad@hotmail.com

* University of Paris. Institute of Psychology. Paris, France. E-mail: merav.dechaume@ parisdescartes.fr - https://orcid.org/ 0000-0002-4817-1744 - External collaborator. E-mail: todd. lubart@parisdescartes.fr - https://orcid.org/ 0000-0002-8776-8797 - President of the non-profit International Society for the Study of Creativity and Innovation (ISSCI). 
Além disso, apoio excessivo à criatividade direta poderia diminuir certos comportamentos relacionados à criatividade, como a fantasia. Estes achados apoiam a noção de que as atitudes dos pais e os comportamentos interativos são preditores significativos das habilidades criativas dos filhos, sugerindo assim possíveis caminhos para pesquisas e práticas educacionais adicionais para apoiar atividades inovadoras e de descoberta.

Palavras-chave: Estilo parental. Modelo multivariado de potencial criativo. Avaliação do potencial criativo.

\begin{abstract}
These days, individuals must be able to think and produce creatively. Therefore, stimulating creative abilities forms an important objective for educators and professionals. Parenting style that includes daily child-rearing practices, creativity specific parent-child interactions, and perceptions about creativity relates fundamentally to the development of creative potential. These constructs shape children's understanding of their environment enabling them to form a mindset, personal qualities, traits and skills that help determine attitudes towards creativity. This preliminary study explored the relationship between parenting style and children's creative potential. Participating parents responded to questionnaires assessing the level of rigidity structure in daily child rearing practices, creativity specific parentchild interactions, and parental perceptions of their children's creative self-efficacy. Their children completed the Evaluation of Creative Potential (EPoC) instrument that measures convergent and divergent thinking in graphic and verbal domains. Although, we found no statistically significant relationship between parenting style and creative potential, several parentchild interactions correlated significantly with parental perceptions. The more parents encourage novelty, the more they perceive their children to show creative behavior. Additionally, too much support for direct creativity could diminish certain creativity - related behaviors such as fantasizing. These findings support the notion that parental attitudes and interactive behaviors are significant predictors of children's creative abilities, thus suggesting possible avenues for further research and educational practices to support novel, discovery activities.
\end{abstract}

Keywords: Parenting style. Multivariate model of creative potential. Evaluation of Creative Potential. 


\section{Estilo parental e potencial criativo de filhos}

A criatividade é uma qualidade essencial para o crescimento socioeconômico pessoal e global, pois os rápidos avanços tecnológicos e o aumento da competitividade exigem a capacidade de pensar e produzir com criatividade (LUBART; BESANÇON; BARBOT, 2019; PUGSLEY; ACAR, 2018). A aquisição de habilidades relacionadas à criatividade como ter mente aberta, persistência e flexibilidade mental, correr riscos e ter curiosidade contribuem para o bem-estar individual e global, tanto no presente quanto para as gerações futuras.

\section{Modelo Quatro-Cs de Criatividade}

A criatividade corresponde à capacidade de produzir novos conteúdos adaptados dentro de seu contexto. Em vez de produzir ideias estranhas ou fora de tópico, a criatividade promove originalidade com significado e valor em seu contexto de produção (LUBART; BESANÇON; BARBOT, 2019). A criatividade se manifesta em quatro níveis de realização. No nível pessoal, a criatividade Mini-c aparece no nível individual quando alguém descobre algo novo e significativo para si mesmo, mas não necessariamente valorizado ou reconhecido por outros. A criatividade $C$ Pequena expande a mini-criatividade no nível pessoal através de feedback e valorização recebidos de outros, como amigos e família. O nível Pró-c oferece originalidade reconhecida por colegas e colaboradores em um ambiente profissional como o mundo literário ou científico. O quarto nível, a criatividade C Grande alcança destaque dentro de um campo através de contribuições significativas reconhecidas mundialmente ao longo do tempo (KAUFMAN; BEGHETTO, 2009).

\section{Modelo Multivariado de Potencial Criativo}

A criatividade guia a evolução da humanidade. Desde os tempos préhistóricos, nossos ancestrais inventaram ferramentas de caça, depois as aperfeiçoaram, uma adaptação para suas necessidades dentro de seu ambiente. 
Os murais encontrados em cavernas, como os de Lascaux, França, ilustram como os indivíduos conseguiram inovar dentro da esfera da comunicação visual (LUBART et al., 2015).

De acordo com a Organização para Cooperação e Desenvolvimento Econômico (ANANIADOU; CLARO, 2009), as crianças precisam dominar um conjunto de habilidades cognitivas, sociais e emocionais que aprimorem a comunicação, a cooperação, o pensamento crítico e a criatividade, a fim de enfrentar os desafios do século XXI. O potencial criativo indica o que um indivíduo pode fazer ao considerar as implicações do ambiente, das experiências de vida e do seu desenvolvimento cognitivo e emocional. O potencial criativo pode mudar e se desenvolver ao longo do tempo, e não é necessariamente uma capacidade criativa geral. Por exemplo, o alto potencial dentro de um domínio científico não necessariamente indica um alto potencial dentro de outro domínio, como a música. O potencial de criação de um indivíduo depende da disponibilidade e acessibilidade a variáveis que, quando combinadas, contribuem para o desenvolvimento do processo criativo (LUBART; BESANÇON; BARBOT, 2019). Esta combinação de componentes representa o principal princípio subjacente ao Modelo Multivariado de Potencial Criativo (ver Figura 1).

FIGURA 1 - MODELO MULTIVARIADO DE POTENCIAL CRIATIVO

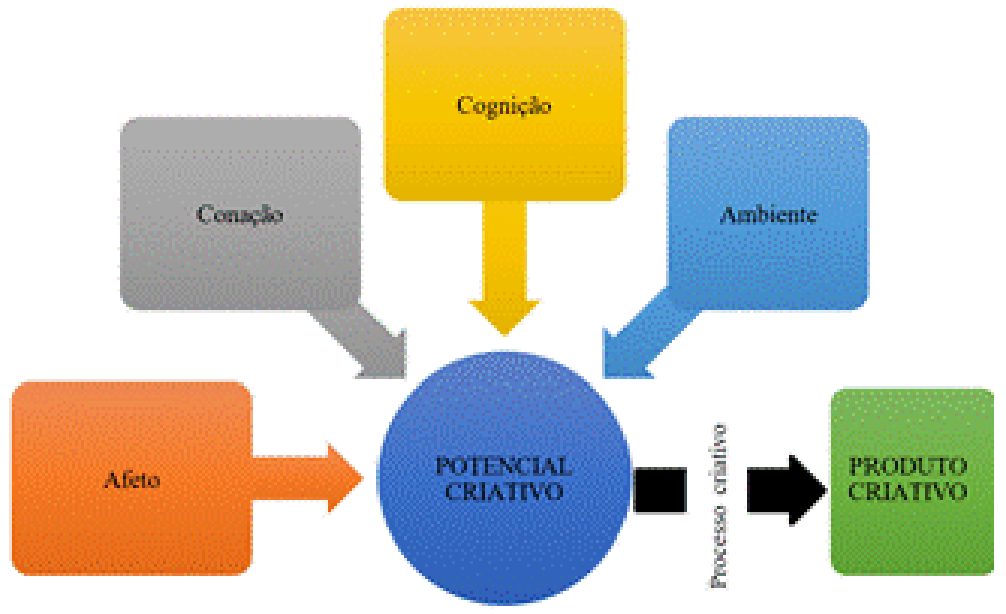

FONTE: Elaborado pelos autores, adaptado de Lubart et al. (2015).

No Modelo Multivariado de Potencial Criativo, os fatores cognitivos referem-se tanto ao conhecimento geral e específico do domínio como à 
capacidade intelectual, como a flexibilidade mental e a capacidade associativa. Entre os fatores positivos estão os traços de personalidade, tais como se arriscar e ter motivação. Fatores afetivos referem-se às qualidades emocionais pessoais, e fatores ambientais consideram o contexto físico e social ao redor do indivíduo (por exemplo, escola, comunidade, família, cultura). Estes componentes contribuem para o potencial de criatividade e estão envolvidos no processo por meio do qual um produto criativo pode ou não ser produzido.

O potencial criativo é uma capacidade latente utilizada durante o envolvimento na realização de uma tarefa. Uma pessoa que não se envolve em uma tarefa não será capaz de mostrar ou utilizar seu potencial e, consequentemente, a produtividade criativa dentro da tarefa. Assim, o envolvimento na realização de uma tarefa se relaciona à motivação, bem como a fatores ambientais que promovem o envolvimento com a tarefa. Por exemplo, o potencial atlético para jogar rúgbi está relacionado às condições ambientais de estatura física e acesso ao treinamento de rúgbi. Portanto, o envolvimento com as tarefas se torna possível quando uma criança cresce fisicamente e ganha acesso às práticas de rúgbi. Caso contrário, o potencial atlético de uma criança como um jogador de rúgbi pode permanecer latente e inexplorado (BESANÇON; LUBART, 2015; STERNBERG; LUBART, 1995).

\section{Medindo o potencial criativo}

A avaliação do potencial criativo pode ocorrer tanto pela medição de componentes individuais quanto dentro de um ambiente avaliativo de testes baseados em cenários sob demandas explícitas (BESANCON; LUBART, 2015). A avaliação componencial considera capacidades generalizadas, específicas de domínio e tarefas específicas, medindo as diferenças individuais. A medição componencial inclui cognição indicada através do nível de flexibilidade mental, conação com traços de personalidade, como abertura a novas ideias, e fatores motivacionais encontrados em escalas e questionários. A abordagem baseada em cenários mede o potencial em vez de uma realização efetiva de fato porque a situação ocorre dentro de um ambiente de testes em vez de dentro de um evento que ocorre naturalmente. A avaliação baseada em cenários mede a capacidade de produção quando os indivíduos se deparam com uma tarefa criativa específica do domínio (por exemplo, artística, escrita, musical) em duas fases distintas, a divergente-exploração e a convergente-integrativa. 
$\mathrm{Na}$ fase de reflexão divergente-exploratória, ocorre um movimento dinâmico entre vários elementos a fim de explorar de forma reflexiva. Na fase divergente-exploratória, um indivíduo transcende os limites de um problema ou tarefa particular e busca novas direções. Esta fase envolve flexibilidade mental, abertura, persistência, curiosidade e motivação, à medida que o indivíduo explora a tarefa dada. A base de conhecimento existente do indivíduo, suas habilidades para investigar e seu ambiente apoiam as funções cognitivas na fase divergente-exploração. A fase convergente-integração apresenta assimilação, agrupamento e tratamento seletivo dos elementos coletados durante a fase divergente-exploratória. O processo seletivo leva à elaboração de uma única ideia ou solução através da convergência e integração (BESANÇON; LUBART, 2015).

\section{$O$ efeito do ambiente familiar}

A produtividade criativa requer apoio ambiental (DENG; WANG; ZHAO, 2016). As relações baseadas na família fornecem a base cognitiva e afetiva à medida que as crianças desenvolvem sua capacidade criativa. $\mathrm{O}$ estilo parental define o ambiente familiar e se refere essencialmente às práticas diárias de educação, atitudes, percepções e comportamentos interativos entre os pais e seus filhos (JANKOWSKA; KARWOWSKI, 2019). O estilo parental ajuda a desenvolver o potencial criativo das crianças, moldando sua compreensão do ambiente em que vivem e sua capacidade de construir mentalidade, qualidades pessoais, traços e habilidades (CROPLEY, 1967; RUNCO; JOHNSON, 2002).

Lautrey (1980) descreveu dois elementos que, quando presentes no ambiente familiar, contribuem para o desenvolvimento intelectual das crianças. Primeiro, as perturbações da vida diária impactam a assimilação do conhecimento e, segundo, um estado de re-equilíbrio proporciona uma eventual construção de novos conhecimentos. Especificamente, Lautrey descreveu o ambiente familiar como uma fonte de perturbação que resiste ao esquema de assimilação piagetiano como uma condição necessária para o re-equilíbrio e, portanto, a eventual construção do conhecimento. Lautrey encontrou estes processos perturbadores evidentes tanto no mundo físico geral quanto no funcionamento familiar diário, com regras de comportamento e hábitos que evocavam uma sensação de regularidade em ambos os ambientes. Esta regularidade permitiu às crianças ver os resultados de suas ações no ambiente familiar cotidiano, como eventos aleatórios semelhantes aos que ocorrem no mundo físico geral. 
Lautrey (1980) descreveu três tipos diferentes de ambientes familiares com base em um continuum de rigidez. Na extremidade mais baixa deste continuum, os eventos perturbadores não são regulados, estruturados e assimilados. As crianças estão em um estado constante de desequilíbrio e, portanto, podem experimentar menos construção cognitiva.

$\mathrm{Na}$ outra ponta do continuum, as crianças vivenciaram ambientes familiares mais previsíveis. As relações entre os eventos pareciam simples, regulares e conhecidas. Embora ambientes previsíveis e estruturados proporcionassem regularidade como condição necessária para a construção cognitiva, não permitiam muitas situações que estimulavam um estado de desequilíbrio. Portanto, as possibilidades de construções cognitivas ocorreram de forma limitada e restritiva. Mais recentemente, alguns pesquisadores fundaram um estilo paternal autoritário ligado a uma tendência de valorizar as características de conformidade e descobriram que um ambiente familiar mais restritivo era preditor negativo do desenvolvimento criativo das crianças (FEARON; COPELAND; SAXON, 2013; TENNET; BERTHELSEN, 1997).

Lautrey (1980) identificou um terceiro ambiente familiar situado entre a baixa rigidez e a alta rigidez nas pontas extremas do continuum. Neste ambiente, a assimilação não estava garantida porque os eventos nem sempre eram regulados da mesma maneira. Neste ambiente do meio, entre o ambiente de baixa rigidez e o de alta rigidez, o indivíduo entrava em um estado de desequilíbrio quando não conseguia relacionar dois eventos previamente integrados. Um novo evento mediador era necessário para a re-assimilação, e eventual construção cognitiva. Estas construções ocorreram através de uma extensão, expansão ou elaboração do esquema inicial.

\section{O propósito do presente estudo}

Em linhas gerais, este estudo preliminar explorou a relação entre o estilo parental e o potencial criativo dos filhos. Examinamos três construtos: comportamentos cotidianos de educação, interações pai-filho específicas da criatividade, e percepções dos pais sobre a autoeficácia criativa de seus filhos. Percebemos esses construtos como importantes fatores que contribuem para o que torna um ambiente familiar criativo. Avaliamos o potencial criativo das crianças através de quatro construtos de realização de tarefas em dois domínios específicos, utilizando a cognição divergente-exploratória e convergenteintegrativa. Por último, determinamos a relação entre cada um dos três construtos de estilo parental com os quatro construtos de potencial criativo das crianças. 


\section{Método}

A metodologia do estudo examinou a relação entre o estilo parental através de uma bateria de três questionários e o potencial criativo das crianças através de uma série de tarefas utilizando o instrumento de Avaliação do Potencial Criativo (EPoC). Os três construtos de estilo parental foram correlacionados com cada um dos quatro construtos que medem o potencial criativo das crianças.

\section{Participantes e procedimentos}

A pesquisadora principal recebeu aprovação para realização do estudo tanto do diretor da escola primária quanto do inspetor regional da Educação Nacional Francesa. Os pais de crianças de 6 a 11 anos de idade que frequentam uma escola primária francesa local em uma pequena vila rural perto de Paris receberam uma carta convite descrevendo o estudo. Sessenta e três das 80 famílias contatadas assinaram e devolveram um termo de consentimento. Os participantes poderiam se retirar do estudo a qualquer momento. Os respondentes receberam um envelope selado com instruções, uma bateria de três questionários autoaplicados totalizando 53 itens, e outro envelope para a devolução dos questionários preenchidos. Os participantes preencheram e devolveram os questionários aos professores. Cada participante representou uma unidade familiar em vez de pais individuais, já que um ou ambos os pais responderam os questionários.

A bateria de estilos parentais incluía itens demográficos básicos. Os participantes das unidades familiares eram predominantemente de nacionalidade francesa ( $98 \%$ dos pais e $95 \%$ das mães). Suas idades variavam de 30 a mais de 45 anos, com uma faixa etária média de 35 a 40 anos, tanto para pais como para mães. A maioria dos pais (70,2\% dos pais; $79,6 \%$ das mães) completou o ensino superior técnico ou acadêmico; alguns (17,5\% dos pais; $15,3 \%$ das mães) completaram apenas o ensino médio. Alguns pais (12,3\%) não completaram o ensino médio e não receberam treinamento em habilidades manuais, e algumas mães $(5,1 \%)$ não completaram o ensino médio. O tamanho da família variou de 1 a 4 filhos, e $88,2 \%$ das famílias relataram ter dois ou mais filhos.

Avaliamos as crianças participantes $(41,4 \%$ meninas; $56,9 \%$ meninos $)$ individualmente em uma sala reservada da escola em duas sessões de teste programadas com uma semana de intervalo. O período de tempo intermediário serviu para neutralizar as flutuações normais nas habilidades criativas dos participantes. O nível escolar das crianças incluiu o primeiro ano $(N=13$; de 6 a 7 anos de idade), o segundo ano $(N=11$; de 7 a 8 anos de idade), o terceiro 
ano $(N=12$; de 8 a 9 anos de idade), o quarto ano $(N=8$; de 9 a 10 anos de idade), e quinto ano $(N=13$; de 10 a 11 anos de idade). Os professores da sala de aula determinaram a ordem de participação individual a fim de minimizar as interrupções no funcionamento diário da escola e no aprendizado das crianças. Das 63 famílias que concordaram em participar, 58 famílias persistiram. Dois pais não preencheram os questionários e um se retirou; duas crianças estavam ausentes para a avaliação do potencial criativo.

\section{Instrumentos}

O estudo utilizou uma bateria de questionários para pais para avaliar o estilo parental e um instrumento para medir o potencial criativo das crianças em idade escolar. $\mathrm{O}$ estilo parental agrupou três construtos incluindo (a) comportamentos parentais diários derivados do instrumento de Lautrey (1980); (b) comportamentos interativos específicos baseados no Questionário Clima para Criatividade na Relação Pais-Criança - CCP-CRQ (KWASENIESKA et al., 2018), e (c) atitudes que refletem itens de autoeficácia criativa baseados na pesquisa de Karwowski et al. (2011). Utilizamos o EPoC durante as duas sessões de avaliação com crianças para gerar oito construtos que medem o potencial de criatividade das crianças.

\section{EpoC}

Utilizamos a versão francesa do $\mathrm{EPoC}$, Avaliação do Potencial Criativo (LUBART; BESANÇON; BARBOT, 2011) para estimar o potencial criativo das crianças. Selecionamos o instrumento EPoC por sua capacidade multidimensional em vez de unidimensional. A maioria das medidas tradicionais de potencial criativo se concentra em tipos divergentes ou convergentes de pensamento criativo. A avaliação realizada utilizando o EPoC envolve as crianças no processo de criação onde ambas produzem muitas ideias a partir de um único estímulo e produzem uma única solução integrando vários elementos. Desta forma, o EPoC mede tarefas divergentes-exploratórias e tarefas convergentesintegrativas em dois domínios familiares às crianças em idade escolar (verbal e gráfico). A avaliação com o EPoC exige que as crianças se reúnam com um avaliador treinado em duas sessões separadas, cada uma com cerca de 45 minutos de duração. A avaliação geral consiste em oito tarefas voltadas para crianças de dois domínios com quatro configurações: divergente-gráfico (DG), divergenteverbal (DV), convergente-integrativa gráfico (IG) e convergente-integrativa verbal (IV). As tarefas DG e DV fornecem pontuação para a fluência com base no número de produções e são referenciadas por normas. As tarefas IV e IG 
são referenciadas por critérios e pontuadas por dois avaliadores independentes e qualificados (BARBOT; BESANÇON; LUBART, 2016). A primeira sessão de avaliação inclui uma breve fase de aquecimento seguida de quatro tarefas. A segunda sessão, aproximadamente uma semana depois, foi composta das quatro tarefas restantes.

\section{Questionários sobre estilo parental}

A bateria de questionários para pais era composta de vários instrumentos. O primeiro conjunto de 20 itens de seleção única de múltipla escolha avaliou um continuum de rigidez expresso em práticas e hábitos diários relacionados à organização do tempo, espaço, atividades físicas e vida social de seus filhos (LAUTREY, 1980). Adaptamos o questionário de 22 itens, removendo 2 itens desatualizados. As respostas não seguiram uma ordem específica na medição do nível de rigidez para evitar possíveis enviesamentos. Por exemplo, a resposta mais rígida poderia aparecer como resposta um, dois ou três com um padrão semelhante para as respostas flexíveis e menos rígidas. Entretanto, todas as respostas mais rígidas receberam um ponto, enquanto o nível mais flexível recebeu dois pontos e as respostas menos rígidas receberam três pontos. As pontuações possíveis variavam de 20 para as respostas mais rígidas a 60 para o nível menos rígido. Um exemplo de um item que media desde o construto menos rígido até o construto mais rígido apareceu como: "Qual é o horário para ir dormir..."

(1) Meu filho vai dormir no mesmo horário todos os dias

(2) Meu filho vai dormir de acordo com o horário que estabelecemos, mas o horário pode variar dependendo da situação (ex.: se não tem aula no dia seguinte) (3) Meu filho vai dormir no horário que quiser

O segundo questionário da bateria explorou as relações pai-filho em relação a comportamentos e ações específicas relacionadas à criatividade. Pedimos a dois pesquisadores bilíngues independentes que traduzissem a versão em inglês do "The Climate for Creativity in Parent-Cild Relationship Questionnaire - CCPCRQ" (KWASENIESKA et al., 2018) para o francês, (com verificação adicional da tradução, de volta para o inglês a partir do francês). Os pais responderam a este conjunto de 23 perguntas de acordo com uma escala de 7 pontos do tipo Likert, variando desde não concordar de forma alguma até concordar fortemente. Estes itens avaliaram os padrões específicos de comportamento dos pais que poderiam moldar a mentalidade das crianças em direção à criatividade. O CCPCRQ incluiu quatro subescalas: incentivo por parte dos pais para experimentar novidade e variedade por exemplo, "Eu mostro ao meu filho a multiplicidade de cores da vida e sua complexidade"; incentivo ao não-conformismo por 
exemplo, "Eu incentivo meu filho com frequência a pensar fora da caixa"; apoio à perseverança nos esforços criativos por exemplo, "Eu mostro ao meu filho a multiplicidade de cores da vida e sua complexidade"), "Quando meu filho tem problemas eu o apoio e o incentivo a considerar soluções diferentes"; e incentivo a fantasiar por exemplo, "Eu falo com meu filho sobre situações e ideias imaginárias engraçadas e estranhas ...”) (KWASENIESKA et al., 2018).

O terceiro questionário mediu as Percepções dos Pais sobre a autoeficácia criativa de seus filhos (KARWOWSKI et al., 2011). Os itens deste questionário avaliaram as opiniões dos pais a respeito das habilidades criativas de seus filhos. Modificamos o questionário original de 11 itens medidos por uma escala de Likert com 4 pontos, removendo um item ambíguo. Nesta medida, os pais responderam aos itens em uma escala de 4 pontos da Likert que variava de nem um pouco a totalmente. Um exemplo deste item do construto afirmava: "Eu acho que meu filho é uma pessoa criativa". Pedimos aos pais que preenchessem o questionário de estilo parental, separadamente ou em conjunto.

\section{Resultados}

As estatísticas descritivas das respostas dos pais para todos os três questionários são apresentadas na Tabela 1. De forma semelhante ao trabalho de Lautrey (1980), formamos três grupos de estrutura de nível de rigidez dos pais participantes. De uma possível faixa de resposta de 20 a 60 pontos, os pais foram identificados como tendo regras rígidas (29-36; $N=22)$, regras flexíveis (37-40; $N=21)$ ou poucas regras $(41-46 ; N=15)$ em seu ambiente doméstico. A baixa confiabilidade deste questionário em nosso estudo $(\alpha=372)$, juntamente com um modelo de 8 fatores que explica apenas $51 \%$ da variação, reflete a natureza independente da maioria dos itens deste questionário.

A medida da interação pai-filho utilizando o CCP-CRQ (KWASENIESKA et al., 2018) em nosso estudo mostrou uma confiabilidade relativamente alta $(\alpha=, 706)$. As subescalas do CCP-CRQ forneceram informações mais detalhadas sobre os tipos e a natureza das interações pai-filho específicas para criatividade. $\mathrm{O}$ intervalo de confidência da subescala foi entre $\alpha=, 735$ ( 6 itens - incentivo ao nãoconformismo) e $\alpha=, 801$ ( 7 itens - apoio à perseverança nos esforços criativos). No terceiro questionário que mede as Percepções dos Pais (KARWOWSKI et al., 2011), os pais classificaram as crianças como mais criativas em vez de menos criativas em suas percepções sobre o eu criativo de seus filhos. A confiabilidade geral deste questionário em nosso estudo foi alta $(\alpha=, 804)$. 
TABELA 1 -ESTATÍSTICAS DESCRITIVAS PARA OS QUESTIONÁRIOS SOBRE ESTILO PARENTAL

\begin{tabular}{l|lllllll}
\multicolumn{1}{c}{} & PR & CC & PP & CC-SPCE & CC-ENC & CC - EENV & CC-EF \\
\hline$N$ & 58 & 58 & 56 & 58 & 58 & 57 & 58 \\
Média & 38,1 & 112 & 29,3 & 6,03 & 4,39 & 5,33 & 1,87 \\
$D P$ & 3,84 & 11,7 & 4,19 & 0,67 & 1,14 & 0,85 & 1,14 \\
Intervalo & 17 & 49 & 18 & 2.71 & 4.67 & 3.57 & 6 \\
Mínimo & 29 & 90 & 20 & 4.29 & 1.83 & 3.29 & 1 \\
Máximo & 46 & 139 & 38 & 7 & 6.5 & 6.86 & 7 \\
Assimetria & -0.09 & 0.23 & 0.05 & -0.48 & -0.45 & -0.51 & 2.85 \\
Erro padr. Assimetria & 0.31 & 0.31 & 0.31 & 0.31 & 0.31 & 0.32 & 0.31
\end{tabular}

Nota. PR - Rigidez Parental, CC - pontuação total para Clima de Criatividade, PP - Percepções Parentais, CC-SPCE - Apoio à Perseverança nos Esforços Criativos, CC-ENC - Incentivo ao Não-Conformismo, CC-EENV - Incentivo a Vivenciar Novidade e Variedade, CC-EF - Incentivo a Fantasiar.

FONTE: Elaborado pelos autores.

A avaliação com o EPoC forneceu dados para examinar a relação entre as pontuações para o potencial criativo das crianças e as pontuações de seus pais para cada um dos três questionários autoaplicados sobre estilo parental. A Tabela 2 apresenta a matriz de correlação $r$ de Pearson utilizada para examinar estes sete construtos.

Não foi possível identificar uma relação entre a pontuação EPoC e os três questionários que compõem nossa bateria de estilo parental. Observamos uma correlação significativa entre a escala do CCP-CRQ (KWASENIESKA et al., 2018) e a escala de Percepções Parentais (KARWOWSKI et al., 2011) $(\mathrm{r}=, 348, \mathrm{p}$ $<, 01)$. Da mesma forma, encontramos uma correlação significativa entre a escala de Percepções Parentais (KARWOWSKI et al., 2011) e a subescala do CCPCRQ (KWASENIESKA et al., 2018) para Incentivo à Vivência da Novidade e Variedade $(\mathrm{r}=, 270, \mathrm{p}<, 05)$. Observamos uma correlação negativa significativa entre a subescala do CCP-CRQ (KWASENIESKA et al., 2018) para Apoio à Perseverança nos Esforços Criativos e Incentivo à Fantasia $(r=-, 315, \mathrm{p}<, 05)$. As intercorrelações entre as tarefas do EPoC, indicaram que os domínios verbal e gráfico não estavam totalmente desconectados e que as pontuações poderiam ser ligadas. Por exemplo, em uma tarefa integrativa-gráfica, as crianças podiam misturar criatividade verbal e gráfica ao explicar sua produção gráfica. 
TABELA 2 -CORRELAÇÃO $R$ DE PEARSON ENTRE CONSTRUTOS DE ESTILO PARENTAL E AVALIAÇÃO DE POTENCIAL CRIATIVO (EPoC)

\begin{tabular}{|c|c|c|c|c|c|c|c|c|c|c|c|}
\hline & PR & $\mathrm{CC}$ & PP & $\begin{array}{c}\text { CC- } \\
\text { SPCE }\end{array}$ & $\begin{array}{l}\text { CC- } \\
\text { ENC }\end{array}$ & $\begin{array}{c}\text { CC - } \\
\text { EENV }\end{array}$ & $\begin{array}{l}\mathrm{CC}- \\
\mathrm{EF}\end{array}$ & DG & DV & IG & IV \\
\hline PR & - & & & & & & & & & & \\
\hline $\mathrm{CC}$ & 0,05 & - & & & & & & & & & \\
\hline PP & 0,18 & $0,35^{* *}$ & - & & & & & & & & \\
\hline CC-SPCE & $-0,13$ & $0,47 * * *$ & 0,13 & - & & & & & & & \\
\hline CC-ENC & 0,16 & $0,68 * * *$ & 0,21 & $-0,11$ & - & & & & & & \\
\hline CC-EENV & 0,1 & $0,77 * * *$ & $0,27^{*}$ & $0,46 * * *$ & 0,24 & - & & & & & \\
\hline CC-EF & $-0,14$ & 0,11 & 0,1 & $-0,31 *$ & 0,06 & $-0,17$ & - & & & & \\
\hline DG & $-0,15$ &,- 05 & 0,03 & $-0,25$ & 0,1 & $-0,02$ & $-0,02$ & - & & & \\
\hline DV & $-0,14$ & 0,04 & 0,2 & $-0,13$ & 0,07 & 0,08 & 0,04 &, 25 & - & & \\
\hline IG & 0,26 & 0,06 & 0,02 & 0,09 & $-0,01$ & 0,07 & $-0,05$ & ,12 & 0,22 & - & \\
\hline IV & 0,14 . & 0,06 & 0,22 & $-0,15$ & 0,18 & 0,01 & 0,04 &, 22 & $0,43 * *$ & $0,333^{*}$ & - \\
\hline
\end{tabular}

$* p<, 05, * * p<, 01, * * * p<, 001$

Nota. PR - Rigidez Parental, CC - pontuação total para Clima de Criatividade, PP - Percepções Parentais, CC-SPCE - Apoio à Perseverança nos Esforços Criativos, CC-ENC - Incentivo ao Não-Conformismo, CC-EENV - Incentivo a Vivenciar Novidade e Variedade, CC-EF - Incentivo a Fantasiar

FONTE: Elaborado pelos autores.

\section{Discussão}

Neste estudo preliminar, exploramos três construtos de estilo parental que podem influenciar o potencial criativo dos filhos. Mais especificamente, consideramos o efeito do nível da estrutura de rigidez parental, exploramos as ligações entre as interações criativas específicas entre pais e filhos e, por último, exploramos as percepções dos pais sobre a autoeficácia criativa de seus filhos. A base conceitual do componente estilo parental indica como os pais mostram seu reconhecimento e expressam seus valores para seus filhos de uma maneira diária e repetitiva. Ao fazer isso, eles moldam qualidades pessoais e constroem habilidades e características que podem promover ou inibir a criatividade por exemplo, Kwasenieska et al. (2018) e Lautrey (1980). Nossa análise não encontrou uma relação estatisticamente significativa entre o desempenho das crianças nas tarefas do EPoC e as avaliações dos níveis de rigidez dos pais. Esta constatação merece uma avaliação mais detalhada de cada item relacionado 
às práticas e hábitos diários entre pais e filhos e uma revisão do construto da estrutura de rigidez parental dentro do sistema de criatividade no ambiente doméstico. Além disso, é interessante avaliar a natureza da relação entre o estilo parental e o potencial criativo. Especificamente, esta relação pode ser descrita como não-linear em vez de uma relação linear, causal?

As pontuações para o questionário CCP-CRQ (KWASENIESKA et al., 2018) não se correlacionaram significativamente com as pontuações para as tarefas do EPoC. Entretanto, as pontuações para o CCP-CRQ (KWASENIESKA et al., 2018) se correlacionaram significativamente com as pontuações para o questionário sobre Percepções Parentais (KARWOWSKI et al., 2011) e, portanto, indicaram semelhanças conceituais em potencial entre o questionário CCP-CRQ (KWASENIESKA et al., 2018) e o questionário sobre Percepções Parentais (KARWOWSKI et al., 2011). Os achados indicaram que as interações pai-filho se correlacionaram significativamente com as percepções dos pais, apoiando assim a noção de que as atitudes e comportamentos interativos dos pais são preditores significativos das habilidades criativas dos filhos (CSIKSZENTMIHALYI, 1996; GUTE et al., 2008; KWASENIEWSKA et al., 2018). É interessante a correlação significativa entre a percepção dos pais sobre a autoeficácia criativa dos filhos e as interações e comportamentos relacionados ao incentivo dos pais para experimentar a novidade e a variedade. Este resultado indica que quanto mais os pais incentivam a novidade, mais eles percebem que seus filhos demonstram comportamento criativo.

Observamos uma correlação negativa significativa entre a subescala de Incentivo à Fantasia do CCP-CRQ (KWASENIESKA et al., 2018) e a subescala de Apoio à Perseverança nos Esforços Criativos do CCP-CRQ (KWASENIESKA et al., 2018). Esta correlação proporcionou uma visão do tipo de comportamento que pode impedir um envolvimento total com a criatividade no ambiente doméstico. Isto pode sugerir que o apoio excessivo à criatividade direta pode diminuir o envolvimento das crianças em comportamentos como a fantasia, ou que a fantasia é muito exigente dentro do contexto da vida diária. Fantasiar exige certas características relacionadas à criatividade, tais como autonomia, independência e questionamento da autoridade e dos valores socioculturais que possivelmente são mais difíceis de adotar diariamente do que a conformidade e o cumprimento (PUGSLEY; ACAR, 2018). 


\section{Conclusão}

A criatividade corresponde à capacidade de produzir novos conteúdos adaptados dentro de seu contexto. O potencial criativo indica o que um indivíduo pode fazer ao considerar as implicações do ambiente, as experiências de vida e o desenvolvimento cognitivo e emocional da pessoa. Os componentes do Modelo Multivariado de Potencial Criativo contribuem para o potencial de criatividade e estão envolvidos no processo através do qual um resultado criativo pode ou não ser produzido. A produtividade criativa requer apoio ambiental. As relações baseadas na família fornecem a base cognitiva e afetiva à medida que as crianças desenvolvem sua capacidade criativa, moldando sua compreensão do ambiente e devido à sua capacidade de construir mentalidade, qualidades pessoais, traços e habilidades. Em linhas gerais, este estudo preliminar explorou a relação entre o estilo parental e o potencial criativo dos filhos.

As correlações relativas às interações entre pais e filhos com as percepções dos pais não permitem determinar um nexo causal. Entretanto, elas sugerem possíveis caminhos para pesquisas e práticas educacionais adicionais para reconhecer e apoiar atividades inovadoras e de descoberta. Começando com os eventos mais essenciais da vida cotidiana, os pais que se envolvem em comportamentos criativos interativos valorizam qualidades relacionadas à autoeficácia criativa, e manter essas competências apoia o potencial criativo de seus filhos. Identificar construtos dentro dos estilos parentais responsáveis pelo desenvolvimento do potencial criativo nos permite aceitar e integrar essas práticas e comportamentos, em nossas famílias, escolas e comunidades. Ao incorporar estes comportamentos no âmbito da família, seremos capazes de reconhecer sua essência e utilidade em um nível social maior.

\section{REFERÊNCIAS}

ANANIADOU, Katrina; CLARO, Magdalean. 21st century skills and competencies for new millennium learners in OECD countries. OECD Education Working Papers, n. 41. Paris: OECD Publishing, 2009. DOI: https://doi.org/10.1787/218525261154. Disponível em: https://www.oecd-ilibrary.org/education/21st-century-skills-and-competences-fornew-millennium-learners-in-oecd-countries_218525261154. Acesso em: 01 nov. 2021.

BARBOT, Baptiste; BESANÇON, Maud; LUBART, Todd. The generality-specificity of creativity: Exploring the structure of creative potential with EPoC. Learning and 
Individual Differences, [S. l.], v. 52, p.178-187, 2016. Disponível em: https://hal. parisnanterre.fr/hal-01392437/file/BBarbot_Revision3.pdf. Acesso em: 11 maio, 2021.

BESANÇON, Maud; LUBART, Todd. La créativité de l'enfant: Évaluation et Développement. Bruxelles: Mardaga, 2015.

CSIKSZENTMIHALYI, Mihaly. Creativity: Flow and the psychology of discovery and invention. New York: Harper Collins Publishers, 1996.

CROPLEY, Arthur. Creativity. London: Longman, 1967.

DENG, Lifang; WANG, Lijuan; ZHAO, Yanyun. How creativity was affected by environmental factors and individual characteristics: A cross-cultural comparison perspective. Creativity Research Journal, Philadelphia, n. 28, p. 357-366, 2016.

FEARON, Danielle; COPELAND, Daelynn; SAXON, Terrill. The relationship between parenting styles and creativity in a sample of Jamaican children. Creativity Research Journal, Philadelphia, n. 25, p.119-12, 2013.

GUTE, Gary et al. The early lives of highly creative persons: The influence of the complex family. Creative Research Journal, Philadelphia, n. 20, p. 343-357, 2008.

JANKOWSKA, Dorota; KARWOWSKI, Maciej. Family factors and development of creative thinking. Personality and Individual Differences, [S. l.], n. 142, p. 202-206, 2019.

KARWOWSKI, Maciej et al. It doesn't hurt to ask... but sometimes it hurts to believe: Polish students' creative self-efficacy and its predictors. Psychology of Aesthetics, Creativity and the Arts, [S. l.], n. 5, p.154-164, 2011.

KAUFMAN, James; BEGHETTO, Ronald. Beyond big and little: The four $\mathrm{c}$ model of creativity. Review of General Psychology, [S. l.], n. 13, p. 1-12, 2009.

KWASENIESKA, Joanna Maria et al. Mothers' personality traits and the climate for creativity they build with their children. Thinking Skills and Creativity, [S. l.], n. 27, p. 13-24, 2018.

LAUTREY, Jacques. Classe sociale, milieu familial, intelligence. Paris: Presses Universitaires de France, 1980.

LUBART, Todd; BESANÇON, Maud; BARBOT, Baptiste. Evaluation du potentiel créatif. Paris: Hogrefe, 2011.

LUBART, Todd et al. Psychologie de la créativité. 2. éd. Paris: Armand Colin, 2015.

LUBART, Todd; BESANÇON, Maud; BARBOT, Baptiste. La créativité, ressource potentielle de l'enfant et l'adolescents, à évaluer, révéler et développer. Neuropsychiatrie de l'enfance et de l'adolescence, [S. l.], v. 67, n. 3, p. 121-129, 2019.

PUGSLEY, Lina; ACAR, Selcuk. Supporting creativity or conformity? Influence of home environment and parental factors on the value of children's creativity characteristics. The Journal of Creative Behavior, [S. l.], v. 54, n. 3, p. 1-12, 2018. DOI: https://doi. 
org/10.1002/jocb.393. Disponível em: https://onlinelibrary.wiley.com/doi/10.1002/ jocb.393. Acesso em: 01 nov. 2021.

RUNCO, Mark; JOHNSON, Diane. Parents' and teachers' implicit theories of children's creativity: A cross-cultural perspectives. Creativity Research Journal, Philadelphia, n. 14, p. 427-438, 2002.

STERNBERG, Robert; LUBART, Todd. Defying the crowd: Cultivating creativity in a culture of conformity. New York: Free Press, 1995.

TENNET, Lee; BERTHELSEN, Donna. Creativity: What does it mean in the family context? Journal of Australian Research in Early Childhood Education, [S. l.], n. 1, p. 91-104, 1997.

Texto recebido em $02 / 05 / 2021$.

Texto aprovado em 23/08/2021. 